\title{
La atención a la diversidad desde la perspectiva de estudiantes del Máster de Educación Secundaria de la Universidad de Jaén
}

\section{Attention to Diversity from the Perspective of Students Doing the Masters in Secondary Education at the University of Jaén}

\author{
M. $^{\text {a }}$ Del Carmen Pegalajar Palomino ${ }^{1}$ \\ mcpegala@ujaen.es \\ Universidad de Jaén, España
}

\section{Resumen:}

El objetivo de este estudio es analizar las percepciones hacia la atención a la diversidad en estudiantes del Máster en Formación del Profesorado de Educación Secundaria $(n=99)$. Para ello se ha utilizado una metodología descriptiva, Ilevándose a cabo la recogida de datos mediante un cuestionario ad hoc. Los resultados revelan cómo el alumnado considera la atención a la diversidad como un deber del centro educativo, capaz de enriquecer a toda la comunidad educativa; manifiesta haber recibido una adecuada formación sobre atención a la diversidad, por lo que se encuentran suficientemente capacitados como para afrontar dicho reto. No obstante, y para éste último aspecto, se aprecian diferencias estadísticamente significativas, siendo más favorables para los estudiantes de la especialidad de Forma-

\begin{abstract}
:
The aim of this study is to analyze the perceptions of students $(n=99)$ doing the Master's degree in Secondary Education regarding attention to diversity. To do this, we used a descriptive methodology, carrying out data collection through an ad hoc questionnaire. The results reveal that students consider attention to diversity is a duty of the school and state that fostering attention to diversity is capable of enriching the whole educational community. The students also highlight they received adequate training on attention to diversity and therefore they perceive they are sufficiently skilled to meet this challenge. However, regarding this latter aspect, statistically significant differences were observed, with better results from students specializing in Education and Guidance than from those specializing in Mathemat-
\end{abstract}

1 Dirección para correspondencia (correspondence address):

M. ${ }^{a}$ del Carmen Pegalajar Palomino. Departamento de Pedagogía. Facultad de Humanidades y Ciencias de la Educación. Universidad de Jaén. Campus "Las Lagunillas", s/n. 23071 Jaén (España) 
La atención a la diversidad desde la perspectiva de estudiantes del Máster de Educación Secundaria de la Universidad de Jaén

M. ${ }^{\mathrm{a}}$ del Carmen Pegalajar Palomino

ción y Orientación Laboral que para los de Matemáticas e Informática.

\section{Palabras clave:}

Profesorado; educación secundaria; diversidad; máster. ics and Computer Science.

\section{Key words:}

Teachers; secondary education; diversity; master's degree.

\section{Résumé:}

L'objectif principal de cette étude est d'analyser les conceptions que se font les étudiants en Master de Formation du professorat pour l'enseignement secondaire, quant à l'attention à la diversité $(\mathrm{n}=99)$. Pour cela, nous avons utilisé une méthode descriptive qui rassemble des données grâce à un questionnaire ad hoc. Les résultats révèlent que les étudiants considèrent l'attention à la diversité comme un devoir de l'école, capable d'enrichir l'ensemble de la communauté éducative. Ils disent également recevoir une formation adéquate sur l'attention à la diversité et sont donc suffisamment formés pour faire face à ce défi. Cependant, des différences significatives ont été observées entre les étudiants de la spécialité de formation et d'orientation de l'emploi, et les étudiants de mathématiques et d'informatique. Ces derniers étant moins favorables à l'attention à la diversité.

\section{Mots clés:}

Les enseignants; I'enseignement secondaire; la diversité; MA.

Fecha de recepción: 05-10-2016

Fecha de aceptación: 09-12-2016

\section{Marco teórico}

La formación inicial del profesorado es un asunto de interés internacional asistido durante la última década a un proceso sostenido de cambios (Tiana, 2013). Una de las reformas más esperadas se relaciona con el Máster Universitario en Profesorado de Educación Secundaria Obligatoria y Bachillerato, Formación Profesional y Enseñanza de Idiomas. El desarrollo de un programa formativo de esta naturaleza debe ofrecer a los futuros profesores unos conocimientos psicopedagógicos y didácticos más profundos, a la vez que ampliar el período de formación de prácticas docentes en centros de secundaria. Además, y coincidiendo con Buendía et al. (2011), la finalidad de esta titulación no es sólo la de capacitar a profesores que resuelvan situaciones educativas sino también, profesionales formados en la reflexión, resolución de problemas, investigación e innovación, que puedan contribuir a que las futuras generaciones de alumnos estén mejor preparadas para afrontar los retos que se les presenten. Además, la formación docente supone un aspecto importante de cara al desarrollo de actitudes positivas (Idol, 2006; Horne 
y Timmons, 2009) y éxito en el desarrollo del proceso inclusivo (Carpenter y Cai, 2011; Jiménez y Hernández, 2013; Mu, Franck y Konz, 2007; Sharma, Moore y Sonawane, 2006).

Tal y como recoge el informe de la UNESCO sobre "Educación para todos" (2014), una de las estrategias clave para la mejora de la institución escolar se basa en optimizar la formación docente para que todo el alumnado pueda aprender. Sin embargo, aún existe la percepción predominante del docente de Educación Secundaria como experto en la didáctica específica (Manso y Martín, 2013), siendo ésta imprescindible tanto como insuficiente. Tal y como se revela en un informe reciente de la OCDE (2012) "Preparing teachers and developing school leaders for the $21^{\text {st }}$ Century", es necesario redefinir los numerosos elementos de los sistemas educativos actuales, pues gran parte de los problemas de la enseñanza en la actualidad se centran en la dificultad de contar con profesores bien preparados. Además, Varcoe y Boyle (2014) destacan la actitud docente como factor importante en el éxito de la educación inclusiva, impulsando la labor desarrollada por las universidades para el desarrollo de las actitudes en el docente en formación.

Valdés, Bolívar y Moreno (2015) entienden cómo la formación inicial del docente debe capacitarlo para hacer realidad el derecho de aprender para toda la población escolar, consiguiendo así la consecución de las competencias clave por parte de todo el alumnado. Así pues, algunos de los estándares formativos necesarios para una enseñanza de calidad se relacionan con el compromiso de docentes hacia el alumnado y su aprendizaje, conocimiento de la materia que enseñan y cómo enseñarla, responsabilidad para gestionar y dirigir el aprendizaje de los estudiantes, reflexión sistemática sobre la práctica y aprendizaje basado en la experiencia e identidad como miembros de comunidades de aprendizaje. Distintas investigaciones (Clotfelter, Ladd y Vidgor, 2007; Gustafsson, 2003; Scheneider y Stern, 2010) ponen de manifiesto la relación que se establece entre la formación del profesorado, sus prácticas y el nivel de logro que alcanza el alumnado. Así pues, y desde la perspectiva inclusiva, las dificultades de aprendizaje son inherentes a todo proceso educativo y, por tanto, su respuesta no debiera considerarse tarea exclusiva de algunos docentes (Duk, 2014). Por ello, resulta prioritario analizar la formación pedagógica de los docentes de Educación Secundaria para así mejorar las prácticas docentes inclusivas en los centros educativos 
y, con ello, el proceso de enseñanza y aprendizaje del alumnado con necesidades específicas de apoyo educativo.

Sánchez y Boix (2008) explican cómo los futuros docentes de Educación Secundaria consideran que la diversidad educativa han de estar presentes en la formación inicial del profesorado de Secundaria; no obstante, admiten tener un desconocimiento absoluto acerca del concepto de atención a la diversidad, factores relacionados con la heterogeneidad educativa, necesidades educativas especiales, estrategias y recursos de integración e inclusión en centros y aulas ordinarias, competencias metodológicas, personales y participativas necesarias para transformar el aula y los entornos de la comunidad de aprendizaje en espacios abiertos al aprendizaje desde el diálogo.

Además, Yanes y Ries (2013) sostienen cómo los estudiantes del Máster en formación del profesorado tienen muy poco conocimiento normativo, teórico y práctico sobre los diversos elementos relacionados con la diversidad; así pues, "un grado de conocimiento adecuado sobre esta dimensión proporcionaría a los futuros docentes herramientas para apoyar específicamente al alumnado con necesidades educativas especiales, con discapacidad y con altas capacidades intelectuales" (Yanes y Ries, 2013, p.117). Conjuntamente, los cambios sufridos en la formación inicial del docente de Educación Secundaria en España, pese al incremento de la formación, ofrecen una capacitación insuficiente al futuro docente para el desempeño de sus funciones como principales agentes de la educación inclusiva (López y Mengual, 2015).

Por el contrario, Alegre (2013) expone cómo los estudiantes del Máster de Formación del Profesorado de Educación Secundaria manifiestan actitudes que se relacionan con el deseo de eliminar barreras hacia la inclusión y la preocupación por mejorar la formación para responder a las peculiaridades del alumnado con necesidades de apoyo educativo. Dichas actitudes han de forjarse desde los primeros momentos de la formación de los futuros docentes, teniendo en cuenta que las mujeres suelen presentar actitudes más favorables hacia la inclusión y que la edad de 28 a 32 años se manifiesta crítica a la hora de considerar las actitudes. De este modo, existe predisposición por parte del alumnado del Máster para fomentar actitudes hacia la inclusión e interculturalidad motivados, principalmente, por la necesidad de que todo el mundo se sienta acogido (culturas inclusivas), apostando por coordinar todas las formas de apoyo para atender la diversidad de los centros (políticas inclusivas) e 
incentivando una filosofía, creencias y valores que defiendan una educación que requiere del aprendizaje de todos: colaboración, participación, cooperación, etc. (Pestano y Alegre, 2013).

Liesa, Arranz y Vázquez (2013) concluyeron cómo una mayor formación respecto a las necesidades específicas de apoyo educativo tiene una repercusión en sus creencias y actitudes, haciéndolas más integradoras. Así, el docente que ha recibido formación específica sobre Educación Especial se muestra más competente en el trabajo con alumnado con necesidades especiales (Hettiarachchi y Das, 2014), pues mejora las habilidades docentes y el proceso de autorreflexión sobre sus capacidades para llevar a cabo esta práctica (Cologon, 2012). En esta misma línea, Pegalajar, Colmenero y Pérez (2013) concluyeron cómo los futuros docentes de Educación Secundaria son conscientes de sus carencias formativas poniendo, a la vez, de manifiesto la necesidad de incorporar dichos aspectos en los planes de estudio de esta titulación, inmersa ya en el proceso de convergencia europea. Algunas de las dificultades y limitaciones propias del título se relacionan con aspectos tales como: poca profundidad en la coordinación interna de las enseñanzas, es decir, entre el módulo genérico de carácter fundamentalmente psicopedagógico y los módulos específicos y el Prácticum; escasa o incorrecta interconexión entre las competencias asignadas a los distintos módulos; y los planes de estudios extremadamente especializados y poco polivalentes desde el punto de vista de su formación didáctico-disciplinar (Valdés y Bolívar, 2014).

Centrados en las especialidades para dicho título, Guisasola, Barragués y Garmendia (2013) exponen cómo el alumnado del Máster de Educación Secundaria adscrito a las especialidades de Ciencias Experimentales, Matemáticas y Tecnología entiende que los contenidos de las áreas educativas incorporadas en el título son interesantes como información, dudando de su utilidad para su trabajo como profesor de ciencias; en cambio, indican acciones concretas vinculadas al establecimiento de relaciones con otros profesores y la organización escolar en el centro. En esta misma línea, Pontes, Serrano y Poyato (2011) sostienen cómo una de las cualidades personales que los futuros profesores de ciencias consideran importantes se relaciona con la actitud hacia el alumnado, subrayando la necesidad de tener en cuenta las características de los estudiantes para tratar de comprender sus dificultades de aprendizaje y a la diversidad del alumnado. 
Por su parte, y para las especialidades más vinculadas al área de Humanidades, Rodríguez, Iñesta y Álvarez-Arregui (2013) reclaman la mejora de la formación del profesorado en aspectos vinculados a la diversidad cultural y lingüística del alumnado, lo que requiere replanteamientos curriculares para trabajar a fondo las actitudes interculturales, el conocimiento y compromiso de respeto a la diversidad sociolingüística, en resumen, el desarrollo de competencias en educación intercultural y plurilingüismo (Cots et al., 2010; González, Guillén y Vez, 2010; Soriano, 2007).

Finalmente, autores como Calvo y Susinos (2010) o Vigo, Soriano y Julve (2010) establecen las directrices de lo que debería incluir un programa de formación inicial del profesorado en el ámbito de la Educación Especial, siendo éste: visión clara de la enseñanza a impartir, currículum fundamentado en la práctica, experiencias clínicas, estándares de la práctica, relaciones entre la Universidad y la escuela, empleo de estudios de caso, indagación y evaluación y análisis reflexivo y crítico (Santos, 2010). De este modo, el docente podrá ofrecer ayudas pedagógicas adecuadas a todo el alumnado una vez cuente con elementos teóricos y prácticos que permitan favorecer el aprendizaje (Sykes, Bird y Kennedy, 2010; Conklin, 2012).

\section{Método}

\subsection{Objetivos}

Esta investigación persigue analizar las percepciones de los estudiantes del Máster en Formación del Profesorado de la Universidad de Jaén hacia la atención a la diversidad en el aula. Así pues, de modo más concreto, se pretende:

- Describir los elementos necesarios para el desarrollo de un proceso de atención a la diversidad de calidad en el aula.

- Analizar el nivel formativo del docente para responder a las necesidades del alumnado de Educación Secundaria.

- Valorar la calidad de la formación recibida por los estudiantes del Máster para responder a los intereses e inquietudes del alumnado con necesidades educativas.

- Analizar las actitudes de los estudiantes hacia la atención a la 
diversidad y la respuesta a las necesidades educativas del alumnado.

- Comprobar la existencia de diferencias estadísticamente significativas en las percepciones de los estudiantes sobre atención a la diversidad según su especialidad.

\subsection{Muestra}

Por su parte, la población objeto de estudio está compuesta por 108 estudiantes del Máster Universitario en profesorado de Educación Secundaria Obligatoria y Bachillerato, Formación Profesional y Enseñanza de Idiomas de la Universidad de Jaén. Para la selección de los sujetos se ha utilizado un muestreo probabilístico aleatorio simple $(\mathrm{n}=99)$. En la tabla 1 se expone, de manera resumida, la distribución de la muestra según variables sociodemográficas estudiadas:

Tabla 1. Caracterización de la muestra.

\begin{tabular}{|c|c|c|c|}
\hline & & $\mathrm{n}$ & $\%$ \\
\hline \multirow{2}{*}{ Género } & Hombre & 39 & 39.4 \\
\hline & Mujer & 60 & 60.6 \\
\hline \multirow{3}{*}{ Edad } & Menos de 25 años & 63 & 63.6 \\
\hline & Entre 26 y 30 años & 16 & 16.2 \\
\hline & Más de 31 años & 20 & 20.2 \\
\hline \multirow{5}{*}{$\begin{array}{l}\text { Titulación de } \\
\text { acceso }\end{array}$} & Ciencias Experimentales & 10 & 10.1 \\
\hline & Ciencias de la Salud & 4 & 4.0 \\
\hline & Enseñanzas técnicas & 18 & 18.2 \\
\hline & Ciencias Sociales y Jurídicas & 7 & 7.1 \\
\hline & Humanidades & 60 & 60.6 \\
\hline \multirow{10}{*}{ Especialidad } & Biología y Geología & 11 & 11.1 \\
\hline & Dibujo, imagen y artes plásticas & 6 & 6.1 \\
\hline & Economía, empresa y comercio & 5 & 5.1 \\
\hline & Formación y orientación laboral & 3 & 3.0 \\
\hline & Ciencias Sociales & 6 & 6.1 \\
\hline & Tecnología y procesos industriales & 11 & 11.1 \\
\hline & Lengua extranjera & 34 & 34.3 \\
\hline & Lengua y literatura & 9 & 9.1 \\
\hline & Matemáticas e Informática & 3 & 3.0 \\
\hline & Orientación educativa & 11 & 11.1 \\
\hline
\end{tabular}




\begin{tabular}{|c|c|c|c|}
\hline & & $\mathbf{n}$ & $\%$ \\
\hline \multirow{7}{*}{$\begin{array}{l}\text { Motivo de } \\
\text { acceso al } \\
\text { Máster }\end{array}$} & Vocación & 45 & 34.6 \\
\hline & Prestigio & 2 & 1.5 \\
\hline & Posibilidad de acceder a un trabajo estable & 63 & 48.5 \\
\hline & $\begin{array}{l}\text { Por no tener una mejor opción en la salida de mi } \\
\text { titulación }\end{array}$ & 4 & 3.1 \\
\hline & $\begin{array}{l}\text { Influencia de algún familiar vinculado a la docen- } \\
\text { cia }\end{array}$ & 8 & 6.2 \\
\hline & $\begin{array}{l}\text { Influencia de algún profesor que me ha marcado } \\
\text { en mi formación }\end{array}$ & 6 & 4.6 \\
\hline & Diversificar la docencia & 2 & 1.5 \\
\hline
\end{tabular}

\subsection{Instrumento de recogida de datos}

El instrumento de recogida de datos utilizado, compuesto de 43 ítems, consiste en una escala tipo Likert cuyas opciones de respuesta oscilan entre 1 y 4 (siendo $1=$ plenamente de acuerdo, $2=$ de acuerdo; $3=e n$ desacuerdo y $4=$ totalmente en desacuerdo). Construida la escala, se procedió a la validación por parte de una docena de expertos universitarios en Pedagogía de la Universidad de Jaén y Granada a los que se les pidió que valoraran y validaran la pertinencia de cada uno de los ítems del cuestionario, estableciendo las consideraciones oportunas para mejorarlo en cuanto a su contenido, ambigüedad, redacción y otros aspectos. Una vez estas sugerencias fueron incorporadas al instrumento, se procedió a la aplicación de una prueba piloto a 90 estudiantes universitarios que se encontraban cursando dicho Máster en otras universidades andaluzas.

Así, el índice de adecuación muestral KMO alcanza un valor de .800 y la prueba de esfericidad de Bartlett es de 3277.395 ( $p=.000)$. Estos datos hacen que se rechace la hipótesis nula de que la matriz de correlación inter-ítems es identidad y se consideran que las respuestas están sustancialmente relacionadas. El análisis de los componentes principales revela, tras la rotación Varimax, la convergencia en 5 factores que explican el $60.80 \%$ de la varianza; los ítems muestran valores apropiados, situándose entre .564 y .853. A continuación, se describe brevemente cada uno de ellos:

- Factor 1: describe los elementos presentes en el proceso de enseñanza y aprendizaje que el docente debe considerar para desarroIlar un proceso de atención a la diversidad de calidad en el aula.

- Factor 2: se centra en analizar el nivel formativo del docente para 
dar respuesta a las necesidades educativas del alumnado en el aula.

- Factor 3: pretende analizar la valoración de los estudiantes del Máster acerca de la formación recibida sobre Educación Especial

- Factor 4: analiza el modo en que la formación recibida permite al futuro docente de Educación Secundaria responder a los intereses e inquietudes detectados en el alumnado con necesidades específicas de apoyo educativo.

- Factor 5: analiza las actitudes de los estudiantes del Máster en Educación Secundaria hacia la atención a la diversidad y respuesta a sus necesidades educativas en el aula en general.

En cuanto a la fiabilidad del instrumento, se ha utilizado el método alpha de Cronbach obteniéndose un valor de .915. Para asegurar aún más la fiabilidad del instrumento, se aplicó el método de las dos mitades, obteniéndose puntuaciones muy apropiadas, pues en la primera parte se obtuvo un valor de .842 y para la segunda el valor obtenido fue de .906 .

\subsection{Procedimiento}

Para el análisis de los datos, se ha utilizado el paquete informático Statistickal Package for Social Sciences (SPSS, v.21) al considerarlo un recurso idóneo para esta investigación. Así pues, se ha llevado a cabo un análisis descriptivo para cada uno de los ítems que componen las escalas del cuestionario. Además, y con la intención de determinar la existencia o no de diferencias estadísticamente significativas en las valoraciones del alumnado acerca del proceso de atención a la diversidad en el aula según la especialidad por la que éstos cursan el Máster en formación del profesorado, se ha realizado un análisis de comparación de medias (ANOVA).

Se destaca como todos los análisis realizados se han calculado con un nivel de confianza del 95\%; asimismo, se ha llevado a cabo un análisis a posteriori a fin de comprobar la diferencia entre todos los pares de medias en el contexto de la muestra total; en concreto, se han asumido varianzas iguales empleando, para ello, la prueba posthoc de Tukey. Al tratarse de un estudio centrado en la Universidad de Jaén, el análisis de varianzas ha asumido diferentes grupos de especialidad, desiguales y de carácter reducido. Se trata de un análisis ANOVA con un diseño des- 
balanceado a causa de la imposibilidad de consecución de suficientes unidades experimentales homogéneas (Díaz, 2009); es decir, no todas las especialidades ofertadas en el Máster cuentan con el mismo número de alumnos matriculados.

\section{Resultados}

En primer lugar, se analizan las puntuaciones de las medias y desviaciones típicas para cada uno de los ítems de los factores que componen el cuestionario. Así pues, para el primer factor "Elementos condicionantes del proceso de atención a la diversidad en el aula", se destaca cómo los estudiantes del Máster consideran la atención a la diversidad como un deber del centro educativo, capaz de enriquecer a toda la comunidad educativa. De igual modo, suponen que la atención a la diversidad requiere de un trabajo colaborativo entre todos los profesionales del centro, de la coordinación entre todos ellos y de la motivación e interés profesional del docente, poniendo de manifiesto cómo la atención al alumnado con necesidades educativas es responsabilidad de todo el profesorado.

Además, se muestran convencidos de que la atención a la diversidad debe ocupar un lugar destacado en su futura práctica docente, siendo una de sus mayores preocupaciones lograr la plena inclusión del alumnado en el aula. Los estudiantes que cursan el Máster de Secundaria consideran que la actitud de la familia supone un aspecto muy influyente en la calidad de la educación del alumnado con necesidades específicas de apoyo educativo. De igual modo, subrayan la labor del Departamento de Orientación o el apoyo de los órganos de dirección del centro en la respuesta a las necesidades educativas del alumnado.

Los encuestados se muestran muy de acuerdo en definir la formación inicial del docente, la disponibilidad de recursos humanos y materiales o una adecuada ratio profesor-alumno como elementos fundamentales para llevar a cabo un proceso de atención a la diversidad de calidad. Se destaca, además, el acuerdo de los encuestados en considerar que la atención a la diversidad pueda llevarse a cabo en todas y cada una de las áreas que componen el currículum de Educación Secundaria. Finalmente, se muestra el alto acuerdo de los estudiantes a la hora de conceder más importancia a la atención a la diversidad en los planes 
de estudio del Máster que están cursando, considerando muy eficaz la combinación de modalidades de escolarización para el alumnado con necesidades educativas entre el aula de apoyo y el aula ordinaria.

\section{Tabla 2. Análisis descriptivo Factor 1.}

\begin{tabular}{lccc}
\hline $\begin{array}{l}\text { Elementos condicionantes del proceso de atención a la diversi- } \\
\text { dad en el aula }\end{array}$ & M. & D.T. \\
\hline Es un deber del centro educativo atender a todo el alumnado & 1.46 & 1,003 \\
\hline $\begin{array}{l}\text { Para una mejor atención a la diversidad se requiere trabajar de } \\
\text { manera colaborativa }\end{array}$ & 1.56 & .883 \\
\hline $\begin{array}{l}\text { La atención a la diversidad de calidad requiere de coordinación } \\
\text { entre el profesorado }\end{array}$ & 1.58 & .938 \\
\hline $\begin{array}{l}\text { La atención a la diversidad de calidad requiere de motivación e } \\
\text { interés profesional }\end{array}$ & 1.61 & .879 \\
\hline $\begin{array}{l}\text { La actitud de la familia influye en la calidad de la educación del } \\
\text { alumnado con n.e.a.e. }\end{array}$ & 1.61 & .924 \\
\hline $\begin{array}{l}\text { La atención al alumnado con n.e.a.e. es responsabilidad de } \\
\text { todo el profesorado }\end{array}$ & 1.63 & .864 \\
\hline $\begin{array}{l}\text { La atención a la diversidad debe ocupar un papel importante en } \\
\text { mi futura práctica docente }\end{array}$ & 1.64 & .942 \\
\hline $\begin{array}{l}\text { Considero fundamental la labor del Departamento de Orienta- } \\
\text { ción en la respuesta a las necesidades educativas }\end{array}$ & 1.68 & .793 \\
\hline $\begin{array}{l}\text { Mi preocupación es que los alumnos logren su inclusión en el } \\
\text { aula }\end{array}$ & 1.70 & .874 \\
\hline $\begin{array}{l}\text { En la atención a la diversidad, el apoyo de los órganos de direc- } \\
\text { ción del centro es prioritario }\end{array}$ & 1.71 & .773 \\
\hline $\begin{array}{l}\text { Un proceso de atención a la diversidad de calidad requiere: } \\
\text { formación inicial sobre atención a la diversidad }\end{array}$ & 1.73 & .831 \\
\hline $\begin{array}{l}\text { Un proceso de atención a la diversidad de calidad requiere de } \\
\text { recursos suficientes y apropiados }\end{array}$ & 1.73 & .843 \\
\hline $\begin{array}{l}\text { La atención a la diversidad en el aula enriquece a toda la comu- } \\
\text { nidad educativa }\end{array}$ & 1.74 & .954 \\
\hline $\begin{array}{l}\text { La atención a la diversidad puede llevarse a cabo en todas y } \\
\text { cada una de las áreas que componen el currículum de Secun- } \\
\text { daria }\end{array}$ & 1.76 & .784 \\
\hline $\begin{array}{l}\text { Un proceso de atención a la diversidad de calidad requiere de } \\
\text { una adecuada ratio profesor-alumno }\end{array}$ & 1.82 & .952 \\
\hline $\begin{array}{l}\text { Se le debería conceder una mayor importancia a la atención a } \\
\text { la diversidad en el Plan de Estudios que estoy cursando }\end{array}$ & 1.96 & .794 \\
\hline $\begin{array}{l}\text { Lo más eficaz para atender la diversidad es combinar la escola- } \\
\text { rización del alumnado con n.e.a.e. entre el aula ordinaria y el } \\
\text { aula de apoyo }\end{array}$ & 1.97 & .839 \\
\hline
\end{tabular}


Para el segundo factor "Respuesta curricular y organizativa a la diversidad en el aula", los estudiantes del Máster de Secundaria manifiestan haber recibido una formación adecuada sobre atención a la diversidad respecto al agrupamiento de los alumnos, selección y adaptación de objetivos, contenidos y competencias básicas, medidas y programas de atención a la diversidad puestos en marcha desde el sistema educativo, organización del espacio, selección, diseño y práctica de actividades y tareas académicas, estrategias metodológicas, evaluación del proceso de enseñanza y aprendizaje y organización del tiempo.

Tabla 3. Análisis descriptivo Factor 2.

\begin{tabular}{lcc}
\hline Respuesta curricular y organizativa a la diversidad en el aula & M. & D.T. \\
\hline Agrupamiento de alumnos & 2.33 & .756 \\
\hline Selección y adaptación de objetivos, competencias y contenidos & 2.40 & .699 \\
\hline Medidas y programas de atención a la diversidad & 2.43 & .758 \\
\hline Organización del espacio & 2.44 & .703 \\
\hline Selección, diseño y práctica de actividades y tareas & 2.45 & .746 \\
\hline Estrategias metodológicas & 2.46 & .760 \\
\hline Evaluación del proceso de enseñanza y aprendizaje & 2.46 & .704 \\
\hline Organización del tiempo & 2.48 & .705 \\
\hline
\end{tabular}

Para el factor "Capacitación docente hacia la diversidad adquirida en el Máster", el alumnado considera que los estudios de Máster realizados les han servido para aumentar su interés hacia la formación de este ámbito, desarrollar una mayor sensibilización hacia la diversidad y para afianzar su elección profesional hacia la docencia. De igual modo, sostienen cómo, tras cursar el módulo genérico del Máster, y más concretamente, la asignatura "Procesos y contextos educativos", han obtenido un conocimiento adecuado sobre la identificación de las necesidades educativas en el alumnado, recursos y servicios prestados desde el sistema educativo, características de los alumnos con necesidades educativas, aspectos básicos del campo de la Educación Especial y legislación sobre el ámbito de la atención a la diversidad. 


\section{Tabla 4. Análisis descriptivo Factor 3.}

Capacitación docente hacia la diversidad adquirida en el Máster $\quad$ M. $\quad$ D.T.

Este Máster me ha servido para aumentar mi interés hacia una mayor formación en el ámbito de la atención a la diversidad

2.19 .888

Este Máster me ha servido para tener una mayor sensibilización hacia la atención a la diversidad

2.19 .900

Este Máster me ha servido para afianzar mi elección profesional hacia la docencia

2.21 .860

Tras cursar el módulo genérico del Máster, poseo un conoci-

miento adecuado sobre la identificación de necesidades educati- $2.29 \quad$ 759

vas en el alumnado

Tras cursar el módulo genérico del Máster, tengo un conocimien-

to adecuado sobre los recursos y servicios prestados desde el

$2.33 \quad .670$

sistema educativo

Tras cursar el módulo genérico del Máster, poseo un conoci-

miento adecuado sobre las características de los alumnos con

$2.34 \quad .717$

necesidades educativas

Tras cursar el módulo genérico del Máster, tengo un conocimien-

to adecuado sobre los aspectos básicos del campo de la Educa-

$2.36 \quad .735$

ción Especial

Tras cursar el módulo genérico del Máster, poseo un conoci-

miento adecuado sobre legislación relativa al ámbito de la

2.43 .688

Educación Especial

En el Máster que estoy cursando he adquirido suficientes conocimientos sobre Educación Especial

Tomando como referencia el factor "Práctica docente formativa en la atención a la diversidad", el alumnado encuestado se muestra favorable en considerar que el proceso de atención a la diversidad requiere de experiencia previa por parte del docente con alumnos con necesidades específicas de apoyo educativo. De igual modo, y tras cursar los módulos genéricos del Máster, se encuentra suficientemente capacitado como para afrontar el reto de la diversidad en el aula, considerando que el desarrollo de actividades y ejemplos prácticos ha mejorado sus conocimientos sobre este campo de estudio, sintiéndose capaces de dar respuesta a las situaciones que se les puedan plantear con alumnado con necesidades específicas de apoyo educativo. No obstante, se encuentran menos convencidos de que el sistema educativo ofrece los servicios y recursos adecuados para atender las necesidades educativas del alumnado escolarizado. 


\section{Tabla 5. Análisis descriptivo Factor 4.}

\section{Práctica docente formativa en la atención a la diversidad}

Un proceso de atención a la diversidad de calidad requiere de experiencia previa con alumnos con necesidades educativas

Me considero suficientemente capacitado como para afrontar el reto de la diversidad en mi aula

Las actividades o ejemplos prácticos desarrollados en las clases han mejorado mis conocimientos sobre atención a la diversidad

Los conocimientos adquiridos sobre atención a la diversidad me darán respuesta a situaciones que se puedan plantear con alumnos con n.e.a.e.

Desde el sistema educativo, se ofrecen los servicios y recursos adecuados para atender las necesidades de los alumnos
M. D.T.

$2.01 \quad .827$

$2.46 \quad .825$

2.51 .761

2.55 .746

Finalmente, para el factor "Percepción docente hacia el alumnado con necesidades específicas de apoyo educativo", los estudiantes del Máster de Educación Secundaria consideran que los alumnos con necesidades específicas de apoyo educativo precisan de numerosas modificaciones en el currículum, lo que supone un trabajo añadido para el profesor. No obstante, se muestran menos favorables en definir como una utopía el hecho de conseguir una adecuada atención a la diversidad en Educación Secundaria, así como en considerar los centros de educación especial como la mejor modalidad de escolarización para el alumnado con necesidades específicas.

Tabla 6. Análisis descriptivo Factor 5.

\begin{tabular}{lcc}
\hline $\begin{array}{l}\text { Percepción docente hacia el alumnado con necesidades específi- } \\
\text { cas de apoyo educativo }\end{array}$ & M. & D.T. \\
\hline $\begin{array}{l}\text { Los alumnos con n.e.a.e. precisan de numerosas modificaciones } \\
\text { en el currículum }\end{array}$ & 2.20 .714 \\
\hline $\begin{array}{l}\text { Trabajar con alumnos n.e.a.e es un trabajo añadido para el profe- } \\
\text { sor tutor en el aula }\end{array}$ & 2.32 .867 \\
\hline $\begin{array}{l}\text { Conseguir una adecuada atención a la diversidad en E.S.O. resul- } \\
\text { ta algo utópico }\end{array}$ & 2.64 .863 \\
\hline $\begin{array}{l}\text { Los alumnos con n.e.a.e. estarían mejor atendidos en centros de } \\
\text { Educación Especial }\end{array}$ & 2.65 .918 \\
\hline
\end{tabular}

Por su parte, el análisis de varianza realizado permite determinar diferencias estadísticamente significativas para algunos de los factores de la escala según la especialidad por la que los estudiantes del Máster cursan 
dicho título de posgrado. En este caso, interesa conocer si el alumnado más afín a especialidades vinculadas a Ciencias Experimentales, Ciencias de la Salud o Enseñanzas técnicas presenta las mismas percepciones hacia el proceso de atención a la diversidad en la etapa de Educación Secundaria que el alumnado cuya especialidades se vinculan al ámbito de las Ciencias Sociales y Jurídicas o Humanidades.

Tabla 7. Análisis de varianza para los factores de la escala según la especialidad.

\begin{tabular}{lccc}
\hline Dimensiones de la escala & ANOVA & Tukey \\
\hline $\begin{array}{l}\text { Elementos condicionantes del proceso de atención a la } \\
\text { diversidad en el aula }\end{array}$ & .941 & - \\
\hline $\begin{array}{l}\text { Respuesta curricular y organizativa a la diversidad en el } \\
\text { aula }\end{array}$ & .250 & - \\
\hline $\begin{array}{l}\text { Capacitación docente hacia la diversidad adquirida en el } \\
\text { Máster }\end{array}$ & $.008^{*}$ & $\mathrm{M} / \mathrm{I}-\mathrm{FOL}$ \\
\hline $\begin{array}{l}\text { Práctica docente formativa en la atención a la diversidad } \\
\text { Percepción docente hacia el alumnado con necesidades } \\
\text { educativas }\end{array}$ & .680 & .890 & - \\
\hline${ }^{*} p<.05$ & &
\end{tabular}

Los resultados obtenidos revelan cómo los estudiantes del Máster en formación del profesorado no muestran diferencias significativas en sus valoraciones hacia el proceso de atención a la diversidad para el factor "Elementos condicionantes del proceso de atención a la diversidad en el aula" $(F(9,89)=.381, p<.941)$. Así pues, independientemente de la especialidad por la que estos alumnos cursan dicho título, las valoraciones hacia los elementos presentes en el proceso de atención a la diversidad en el aula son favorables.

En segundo lugar, y para el factor "Respuesta curricular y organizativa a la diversidad en el aula", tampoco se aprecian diferencias estadísticamente significativas en sus resultados $(F(9,89)=1.298, p<.250)$. De este modo, las percepciones del alumnado del Máster hacia su nivel formativo para dar respuesta a las necesidades educativas del alumnado en el aula son positivas, independientemente de la especialidad por la que cursan este título de posgrado.

Relacionado con su formación, los resultados demuestran cómo tampoco existen diferencias estadísticas significativas según la especialidad del alumnado para los factores "Práctica docente formativa en la 
atención a la diversidad" $(F(9,89)=.730, p<.680)$ y "Percepción docente hacia el alumnado con necesidades específicas de apoyo educativo" $(F(9,89)=.471, p<.890)$. Así pues, se aprecian unas las actitudes y valoraciones favorables hacia la respuesta educativa de los futuros docentes a los intereses e inquietudes del alumnado con necesidades específicas de apoyo educativo en el aula.

Sin embargo, existen diferencias estadísticamente significativas en las valoraciones del alumnado para el factor "Capacitación docente hacia la diversidad" según su especialidad $(F(9,89)=2.703, p<.008)$. En este caso, y tal y como aparece en el gráfico adjunto, los alumnos que cursan la especialidad de Formación y Orientación Laboral muestran unas valoraciones más favorables acerca de la formación recibida en el Máster hacia la atención a la diversidad $(M=16.00 ; \mathrm{DT}=6.24)$ que aquellos otros que lo hacen por la especialidad de Matemáticas e Informática $(M=28.33$; DT=5.85).

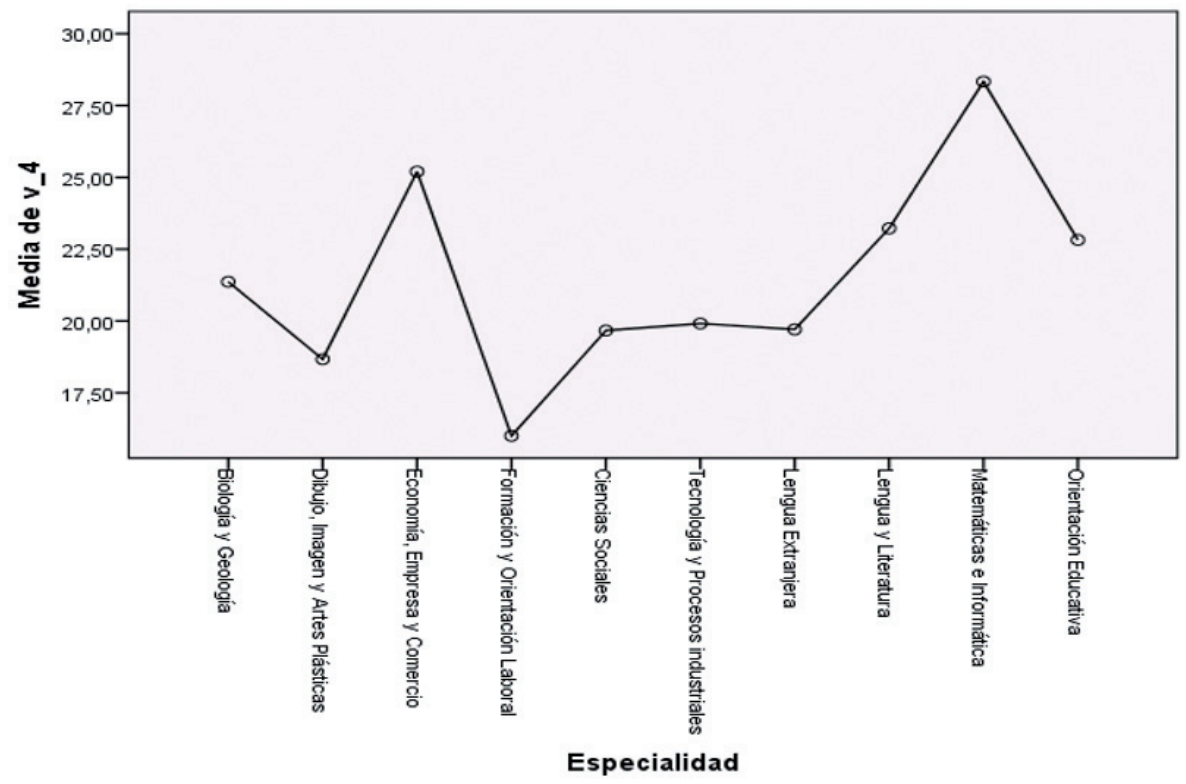

Figura I. Puntuaciones medias para la dimensión "Capacitación docente hacia la diversidad". 


\section{Discusión de resultados y conclusiones}

Este estudio pretende analizar las percepciones de los estudiantes del Máster de Formación del Profesorado de la Universidad de Jaén hacia la atención a la diversidad. Así pues, los encuestados entienden que el proceso de atención a la diversidad en el aula requiere del trabajo colaborativo entre todos los profesionales del centro, coordinación entre todos ellos así como motivación e interés hacia la diversidad. Además, se precisa experiencia previa con alumnado con necesidades educativas por parte del docente, mostrándose suficientemente capacitado como para afrontar el reto de la diversidad en el aula. La respuesta educativa que se le debe ofrecer a este tipo de alumnado supone desarrollar numerosas modificaciones en el currículum, siendo éste un trabajo añadido para el profesor. Estos resultados coinciden con las conclusiones expuestas por Varcoe y Boyle (2014) quiénes aluden a la actitud docente como factor importante en el éxito de la educación inclusiva. Además, Duk (2014) subraya cómo las necesidades educativas del alumnado son inherentes al proceso educativo, no debiendo llevarse a cabo su respuesta educativa sólo por parte de algunos docentes.

Además, el alumnado participante en la investigación considera que la formación recibida sobre aspectos curriculares y organizativos hacia la diversidad es la adecuada en cuestiones vinculadas al agrupamiento del alumnado, selección y adaptación de objetivos, contenidos y competencias básicas, medidas y programas de atención a la diversidad ofrecidos desde el sistema educativo español, organización espacio-temporal, estrategias metodológicas y diseño de actividades y su posterior evaluación. Estos datos contradicen los aportados por Sánchez y Boix (2008) quiénes analizan el bagaje previo de los aspirantes a profesores de Educación Secundaria; así pues, se demuestra el desconocimiento del alumnado que asiste al Curso de Aptitud Pedagógica acerca del concepto de atención a la diversidad, estrategias y recursos de integración e inclusión, competencias metodológicas y entornos de comunidad de aprendizaje. De igual modo, Yanes y Ries (2013) demostraron el poco conocimiento normativo, teórico y práctico de los estudiantes del Máster sobre elementos relacionados con la diversidad. Más recientemente, López y Mengual (2015) consideran cómo los cambios en la formación inicial del docente de Educación Secundaria resultan insuficientes para capacitarle 
hacia el desempeño de sus funciones como principales agentes de la educación inclusiva.

Centrados en la formación recibida en sus estudios de posgrado, los estudiantes consideran que dicho Máster ha supuesto una oportunidad para aumentar su interés hacia la formación en el ámbito de la Educación Especial, mayor sensibilización para la diversidad y afianzamiento de su elección profesional hacia la docencia. Estos resultados guardan relación con las ideas expuestas por Alegre (2013), quién manifiesta la preocupación de los estudiantes del Máster en Formación del Profesorado para mejorar su formación para responder a las necesidades educativas del alumnado. De igual modo, Liesa, Arranz y Vázquez (2013) muestran cómo la formación docente sobre atención a la diversidad repercute de manera positiva en sus creencias y actitudes hacia las necesidades educativas del alumnado, mostrándose más competente en su práctica profesional (Hettiarachchi y Das, 2014). Sin embargo, y tal y como muestra Reoyo et al. (2012) dicha formación debe aglutinar tanto aspectos curriculares como afectivos y desarrollo de habilidades relacionales.

Según la especialidad por la que el alumnado cursa el Máster en formación del profesorado, los resultados demuestran cómo se producen diferencias a nivel estadístico entre el alumnado que cursa dicho título para la especialidad de Matemáticas e Informática y aquellos otros que lo hacen para Formación y Orientación Laboral. En este caso, el alumnado afín a las especialidades de Formación y Orientación Laboral se muestra más capacitado hacia la diversidad que aquel adscrito a la especialidad de Matemáticas e Informática. Estos datos guardan relación con los aportados por Guisasola, Barragués y Garmendia (2013) quiénes demuestran cómo el alumnado del Máster para las especialidades de Ciencias Experimentales, Matemáticas y Tecnología considera que los programas de formación inicial del docente contienen contenidos interesantes como formación, aunque dudan de su utilidad para su profesión como docente del área de ciencias. Así pues, uno de los alumnos comentaba cómo "...el módulo de Pedagogía me ha permitido conocer el contexto de la educación secundaria... sin embargo, he echado en falta el análisis de casos concretos que pueden sucederme cuando empiece a dar clase... En las prácticas me ha tocado un centro en el que la profesora tenía graves problemas de disciplina en el aula... Sobre esto no hemos comentado nada en el módulo genérico y sólo en una materia 
de didáctica específica se ha comentado lo importante que es interesar e implicar al alumnado". (p. 575).

Ello impide analizar las características de los estudiantes con necesidades educativas, tratando de comprender sus dificultades de aprendizaje así como la diversidad del alumnado (Pontes, Serrano y Poyato, 2013). No obstante, Rodríguez, Iñesta y Álvarez-Arregui (2013), para el área de Humanidades, demuestra la necesidad de mejora en la formación del profesorado para aspectos vinculados a la diversidad cultural y lingüística del alumnado.

Sin embargo, y tal como apuntan León y Arjona (2011): "si bien en la realidad actual de los centros de secundaria en el camino hacia la inclusión existen elementos que lo obstaculizan, es alentador pensar que, en la mayor parte de los aspectos analizados, el profesorado ve la necesidad de cambio y de superación" (p.221).

En definitiva, y a modo de síntesis, se evidencia la buena predisposición de los futuros docentes de Educación Secundaria hacia el desarroIlo de un proceso de atención a la diversidad de calidad, capaz de lograr la plena inclusión del alumnado con necesidades específicas de apoyo educativo en el aula. El Máster es concebido, desde este punto de vista, como una nueva oportunidad formativa para adquirir conocimientos didácticos y psicopedagógicos más profundos que les capacite para su futuro ejercicio profesional en el aula (Buendía et al., 2011).

Este trabajo pretende conocer las valoraciones de los estudiantes del Máster en Formación del Profesorado hacia el proceso de atención a la diversidad para así establecer mejoras en el plan de estudios que respondan a las exigencias planteadas. Su interés reside en conocer no sólo las percepciones del futuro docente de Educación Secundaria hacia la diversidad, sino las concepciones acerca de las potencialidades de la formación para su futura práctica docente. No obstante, la escasa muestra con la que se ha llevado a cabo dicha investigación no nos permite garantizar la generalización de los resultados a otras muestras. Además, el uso exclusivo del cuestionario como instrumento de recogida de datos puede generar problemas de deseabilidad social y sinceridad al cumplimentarlo. De cara a futuras investigaciones, podría resultar interesante comparar las percepciones sobre atención a la diversidad entre los estudiantes del Máster de Secundaria y los docentes que se encuentran en activo en la etapa de Educación Secundaria Obligatoria, examinando si dichas valoraciones se modifican o sufren ligeros cambios tras la práctica de su ejercicio profesional. 
La atención a la diversidad desde la perspectiva de estudiantes del Máster de Educación Secundaria de la Universidad de Jaén

M. a del Carmen Pegalajar Palomino

\section{Referencias bibliográficas}

ALEGRE, O. M. (2013). La formación del profesorado de Educación Secundaria en competencias vinculadas a la inclusión: el caso de la Universidad de La Laguna. En S. Castillo (coord.). El profesor de Educación Secundaria para el siglo XXI. Fundamentos para su formación (pp. 31-44). Universidad Nacional de Educación A Distancia: Madrid.

BuendíA, L., BeRROCAL, E., OlMEDO, E., PEGALAJAR, M., RUIZ, M. y TOMÉ, M. (2011). Valoración por parte del alumnado de las competencias que se pretenden conseguir con el Máster Universitario de Profesorado en Educación Secundaria Obligatoria, Bachillerato, Formación Profesional y Enseñanza de Idiomas. Bordón. Revista de Pedagogía, 63 (3), 57-74.

CALVO, A. y SUSINOS, T. (2010). Prácticas de investigación que escuchan la voz del alumnado: mejorar la universidad indagando la experiencia. Profesorado. Revista de Curriculum y formación del profesorado, 14 (3), 1-14.

CARPENTER, C. y CAI, S. (2011). Effect of clinic experience on pre-service professionals perceptions of applied special needs services. Revista Brasileira de Cineantropometria \& Desempenho Humano, 13(2), 145-149.

CLOTFELTER, C.T; LADD, H.F. y VIDGOR, J.L. (2007). Teacher credentials and student achievement in High School: A Cross-subject analysis with student fixed effects. Disponible en: http://www.nber.org/papers/w13617.pdf?new_window=1 (consultado el 15 de Septiembre de 2016).

COLOGON, K. (2012). Confidence in their own ability: Postgraduate early childhood students examining their attitudes towards inclusive education. International Journal of Inclusive Education, 16(11), 1155-1173.

CONKLIN, H. (2012). Tracing learning from divergent teacher education pathways into practice in middle grades classrooms. Journal of Teacher Education, 63(3), 171-184.

COTS, J.M., IBARRARAN, A., IRÚN, M., DASAGABASTER, D., LLURDA, E y SIERRA, J.M. (2010). Plurilingüismo e interculturalidad en la escuela. Reflexiones y propuestas didácticas. Barcelona: Horsori.

DíAZ, A. (2009). Diseño estadístico de experimentos. Colombia: Universidad de Antioquía.

DUK, C. (2014). La formación y el desarrollo profesional de los docentes para una educación inclusiva. En Marchesi, A., Blanco, R. y Hernández, L. (coords.) Avances y desafíos de la educación inclusiva en Iberoamérica (pp. 61-71). Madrid: OEI.

GONZÁLEZ, M., GUILLÉN, C. y VEZ, J.M. (2010). Didáctica de las lenguas modernas: competencia plurilingüe e intercultural. Madrid: Síntesis.

GUISASOLA, J., BARRAGUÉS, J. I., y GARMENDIA, M. (2013). El Máster de Formación Inicial del Profesorado de Secundaria y el conocimiento práctico profesional del futuro profesorado de Ciencias Experimentales, Matemáticas y Tecnología. Revista Eureka sobre Enseñanza y Divulgación de las Ciencias, 10, 568-581.

GUSTAFSSON, J.E. (2003). What Do We Know About Effects of School Resources on Educational Results? Swedish Economic Policy Review, 10, 77-110.

HETTIARACHCHI, S. y DAS, A. (2014). Perceptions of inclusion and perceived prepa- 
La atención a la diversidad desde la perspectiva de estudiantes del Máster de Educación Secundaria de la Universidad de Jaén M. a del Carmen Pegalajar Palomino

redness among school teachers in Sri Lanka. Teaching and Teacher Education, 43, 143-153.

HORNE, P. y TIMMONS, V. (2009). Making it work: teachers perspectives on inclusion. International Journal of Inclusive Education, 13(3), 273-286.

IDOL, L. (2006). Toward inclusion of special education students in general education. A program evaluation of eight schools. Remedial and Special Education, 27(2), 77-94.

JIMÉNEZ, L. y HERNÁNDEZ J.L. (2013). Initial training for inclusive physical education: situation, prospects and competencies. Revista Internacional de Medicina y Ciencias de la Actividad Física y del Deporte, 13(51), 471-494.

LEÓN, M.J. y ARJONA, Y. (2011). Pasos hacia la inclusión escolar en los centros de Educación Secundaria Obligatoria. Revista de Innovación Educativa, 21, 201-211.

LIESA, M., ARRANZ, P. y VÁZQUEZ, S. (2013). Un programa basado en la metodología del aprendizaje servicio que mejora las actitudes de los estudiantes del Grado de Magisterio hacia la inclusión. Revista Interuniversitaria de Formación del Profesorado, $76(27,1), 65-82$.

LÓPEZ, M. y MENGUAL, S. (2015). Un ataque a la educación inclusiva en la Educación Secundaria. Limitaciones de la formación inicial del profesorado en España. New Approaches in Educational Research, 4, 10-18.

MANSO, J. y MARTíN, E. (2013). Valoración del Máster de Formación del Profesorado de Educación Secundaria: estudio de casos en dos universidades. Revista de Educación, 364, 145-169.

MU, K., FRANCK, L. G. y KONZ, C. (2007). Attitudes of entry level occupational therapy doctoral students towards inclusion for students with disabilities. Australian Occupational Therapy Journal, 54, 66-73.

PEGALAJAR, M.C.; COLMENERO, Mª .J. y PÉREZ, E. (2013). Necesidades formativas del profesorado de educación secundaria sobre atención a la diversidad. En S. Castillo (coord.). Reflexiones, Análisis y Propuestas sobre la Formación del Profesorado de Educación Secundaria (pp. 118-120). Universidad Nacional de Educación A Distancia: Madrid.

PESTANO, M.A. y ALEGRE, O.M. (2013). La formación del profesorado de Educación Secundaria en actitudes hacia la inclusión e interculturalidad de la universidad de La Laguna. En S. Castillo (coord.). Reflexiones, Análisis y Propuestas sobre la Formación del Profesorado de Educación Secundaria (pp. 164-167). Universidad Nacional de Educación A Distancia: Madrid.

PONTES, A.; SERRANO, R. y POYATO, F.J. (2013). Concepciones y motivaciones sobre el desarrollo profesional docente en la formación inicial del profesorado de educación secundaria. Revista Eureka sobre Enseñanza y Divulgación de las Ciencias, 10, 533-551.

REOYO, N., CARBONERO, M.A., FREITAS, A. y VALDIVIESO, J.A. (2012). La percepción de los futuros profesores sobre los docentes de Educación Secundaria. International Journal of Developmental and Educational Psychology INFAD Revista de Psicología, 1(2), 389-396.

RODRÍGUEZ, A., IÑESTA, E.M y y ÁLVAREZ-ARREGUI, E. (2013). Diversidad cultural y plurilingüismo en Asturias: un estudio empírico sobre las percepciones del futuro profesorado. European Journal of Education and Psychology, 6(2), 117-133. 
La atención a la diversidad desde la perspectiva de estudiantes del Máster de Educación Secundaria de la Universidad de Jaén

M. a del Carmen Pegalajar Palomino

SÁNCHEZ, A. (2007). Investigación sobre la formación inicial del profesorado de educación secundaria para la atención educativa a los estudiantes con necesidades educativas. Revista Interuniversitaria de formación del profesorado, 21 (2), 149-181.

SÁNCHEZ, A. y BOIX, J.L. (2008). Los futuros profesores de educación secundaria: inicio de su profesionalización y construcción de su identidad docente. Revista Electrónica Interuniversitaria de Formación del profesorado, 11 (2), 31-45.

SANTOS, M.A. (2010). La formación del profesorado en las instituciones que aprenden. Revista Interuniversitaria de Formación del Profesorado, 68 (24,2), 175-200.

SCHNEIDER, M. y STERN, E. (2010). The cognitive perspective on learning: ten cornerstone findings. En Organisation for Economic Co-Operation and Development (OECD) (Ed.). The nature of learning: Using research to inspire practice (pp. 69-90). Paris: OECD.

SHARMA, U., MOORE, D. y SONAWANE, S. (2009). Attitudes and concerns of preservice teachers regarding inclusion of students with disabilities into regular schools in pune, India. Asia-Pacific Journal of Teacher Education, 37(3), 319-31.

SORIANO, E. (2007). Educación para la convivencia intercultural. Madrid: La Muralla.

SYKES, G., BIRD, T. y KENNEDY, M. (2010). Teacher education: Its problems and some prospects. Journal of Teacher Education, 61, 464-476.

TIANA, A. (2013). Los cambios recientes en la formación inicial del profesorado en España: una reforma incompleta. Revista Española de Educación Comparada, 22, 39-58.

UNESCO. 2014. Enseñanza y aprendizaje: lograr la calidad para todos. París: UNESCO. http://unesdoc.unesco.org/images/0022/002261/226159s.pdf (consultado el 7 de Septiembre de 2016).

VALDÉS, R. y BOLÍVAR, A. (2014). La experiencia española de formación del profesorado: el Máster en Educación Secundaria. Ensino Em Re-Vista, 21, 159-173.

VALDÉS, R., BOLÍVAR, A. y MORENO, A. (2015). Una valoración de la formación inicial de profesores en España: El Máster en Educación Secundaria. Educaçao em Revista, 31, 251-278.

VARCOE, L. YyBOYLE, C. (2014). Pre-service primary teachers`attitudes towards inclusive education. Educational Psychology, 34(3), 323-337.

VIGO, B., SORIANO, J. y JULVE, C. (2010). Preparando profesores para la atención a la diversidad: potencialidades y limitaciones de un proyecto de innovación y mejora interdisciplinar. Revista Interuniversitaria de Formación del Profesorado, 69(24,3), 147-165.

YANES, C. y RIES, F. (2013). Liderando el cambio: estudios sobre las necesidades formativas de los futuros docentes de Secundaria. Revista Fuentes, 14, 105-124. Disponible en: http://www.revistafuentes.es/ (consultado el 26 de Septiembre de 2016). 